Article

\title{
Linear System Identification and Vibration Control of End-Effector for Industrial Robots
}

\author{
Xiaobiao Shan ${ }^{1}$, Henan Song ${ }^{1,+} \oplus$, Chong Zhang ${ }^{1}$, Guangyan Wang ${ }^{2}$ and Jizhuang Fan ${ }^{1, *}$ \\ 1 State Key Laboratory of Robotics and System, Harbin Institute of Technology, Harbin 150001, China; \\ shanxiaobiao@hit.edu.cn (X.S.); 19B908033@stu.hit.edu.cn (H.S.); 17S008004@stu.hit.edu.cn (C.Z.) \\ 2 Hefei Sineva Intelligent Machine Co., Ltd., Hefei 230011, China; wangguangyan@sineva.com.cn \\ * Correspondence: fanjizhuang@hit.edu.cn; Tel.: +86-1383-619-5902 \\ + Current address: 2F, Science Park, Harbin Institute of Technology, Nangang District, Harbin 150001, China.
}

Received: 11 November 2020; Accepted: 27 November 2020; Published: 29 November 2020

check for updates

\begin{abstract}
This paper presents the discrete state space mathematical model of the end-effector in industrial robots and designs the linear-quadratic-Gaussian controller, called LQG controller for short, to solve the low frequency vibration problem. Though simplifying the end-effector as the cantilever beam, this paper uses the subspace identification method to determine the output dynamic response data and establishes the state space model. Experimentally comparing the influences of different input excitation signals, Chirp sequences from $0 \mathrm{~Hz}$ to $100 \mathrm{~Hz}$ are used as the final estimation signal and the excitation signal. The LQG controller is designed and simulated to achieve the low frequency vibration suppression of the structure. The results show that the suppression system can effectively suppress the fundamental natural frequency and lower vibration of end-effector. The vibration suppression percentage is $95 \%$, and the vibration amplitude is successfully reduced from $\pm 20 \mu \mathrm{m}$ to $\pm 1 \mu \mathrm{m}$. The present work provides an effective method to suppress the low frequency vibration of the end-effector for industrial robots.
\end{abstract}

Keywords: linear system identification; low frequency vibration; end-effector

\section{Introduction}

The problem of vibration suppression has traditionally been a research hotspot of scholars, and studies on vibration control are increasing year by year [1]. Due to the advantages of lightweight, large output, fast response speed and high strain sensitivity, piezoelectric materials have been widely used for vibration control and other occasions [2]. What is more, a piezoelectric patch can be easily attached to the cantilever structure which is the most used mechanism in the area of industrial robots and the most easily simplified model for transportation robots [3,4]. Therefore, using piezoelectric materials for vibration suppression of beam structures has been widely studied.

Piezoelectric material was introduced into the beam vibration by Crawley and Luis [5] for the first time, and subsequently, it was widely used in robot structure. Some scholars use the positive piezoelectric effect and the inverse effect to design sensor and actuator for robot. It achieved the collection of the vibration signal through a sensor and gave a reverse vibration by the actuator in order to achieve the mechanism vibration suppression, e.g., Tzou et al. [6,7], Shen et al. [8], Lin [9] and Lou [10]. However, soon the hysteresis characteristic of piezoelectric materials and the interference between electrical signals appeared. Leang [11] found the influence of the time-delay effect of piezoelectric materials on vibration suppression and started to study the hysteresis characteristics, as well as Zhang [12], Chen [13], and so on. In addition, some scholars focused on separation of control signals.

Another research direction is only using piezoelectric materials as actuators. Dadfarnia [14] used a piezoelectric (PZT) patch as actuator which is bonded on the surface of the flexible beam to suppress 
residual vibration. Qiu et al. [15] gave a vibration suppression method of two bending modes and two torsional modes. At the background of this research direction, Chang et al. [16] discussed using an auxiliary piezoelectric actuator to control vibration for high-speed linear robots. Because of the minute output of piezoelectric material, it is often used in many pieces to achieve the precise vibration suppression of robots. Jia et al. [17], Zehetner [18] and Douat et al. [19]. Yang et al. [20] studied the accurate models of a flexible link and two surfaces bonded with piezoelectric patches, where the link and the piezoelectric patches will be modelled through the use of Euler-Bernoulli beam theory (EBT).

When using PZT patch as actuator, in order to achieve vibration suppression, some people have studied the model identification of the system. Narendra [21] carried out detailed research on system identification and real-time control of dynamic systems, and realized adaptive control of nonlinear systems. People of the same range studied the identification method based on dynamic system, e.g., Abd Jalil [22], Sethi [23-25]. Elsley [26] of Rockwell International Science Center established a self-learning double-layer back propagation (BP) neural network control system for unknown characteristics and dynamic model systems, while Song [27] established neural network discrimination. Chen et al. [28,29] studied an adaptive method and gave the relationship between the proportion of low frequency components and the modal order. Some identification models came from experiments, such as Takawa et al. [30].

The existing research on vibration control of beam structure lacks the research on industrial robot with low frequency vibration. At the same time, under this working condition, most adaptive control systems or feedback compensation systems cannot provide the feedback signals needed by the system in real time. Therefore, a vibration controller based on linear system identification is proposed to solve the problem of low frequency oscillation.

The remainder of this paper is organized as follows. The linear system identification and LQG controller of the structure of cantilever beam are described in Section 2. In Section 3, the linear identification system is designed. Furthermore, the LQG controller is presented in Section 4. Conclusions are shown in Section 5.

\section{Materials and Methods-Structure Design of Cantilever Beam}

Since the first generation of industrial robots came out in 1945, the application of industrial robots has gradually become more and more widespread [31]. More and more industrial robots replace human to complete industrial tasks with unusual or difficult requirements. People have higher and higher requirements for the quality of industrial robots. This paper mainly studies the transport robot for glass substrate shown in Figure 1. Due to the heavy weight, large area and easy damaging of the glass substrate, the position accuracy of the end-effector of the transport robot is required to be high. In practice, the vibration of the end-effector is the lead cause of glass substrate damage. Therefore, based on the end-effector of a glass substrate handling robot, a cantilever structure is designed for system identification and vibration control.

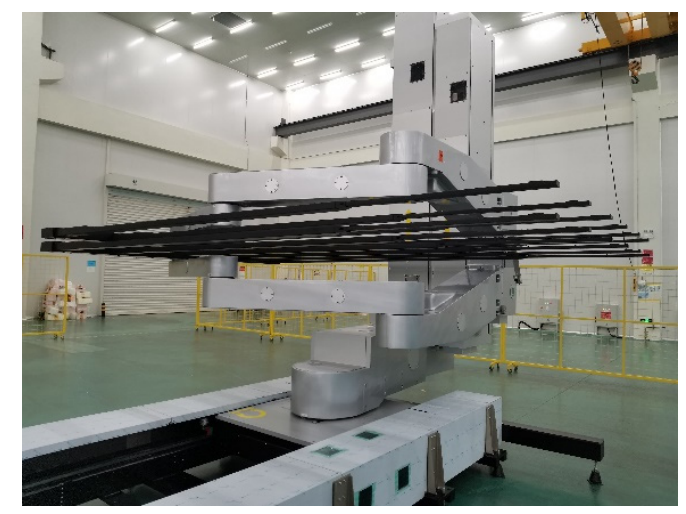

Figure 1. Glass transport robot. 
The end execution structure of the existing glass substrate handling robot is illustrated in Figure 2. The overall structure is a rod-shaped structure with uniform mass distribution, large span and relatively thin thickness. The upper surface is the contact surface of the glass substrate which is connected by the glass substrate absorber. The end-effector is usually fixed to the arm by riveting or screw insertion into the arm mounting hole. Most substrates are composed of rubber while the material of the end-effector is steel and carbon fiber. Therefore, the structure can be simplified as a cantilever beam model, as shown in Figure 3.

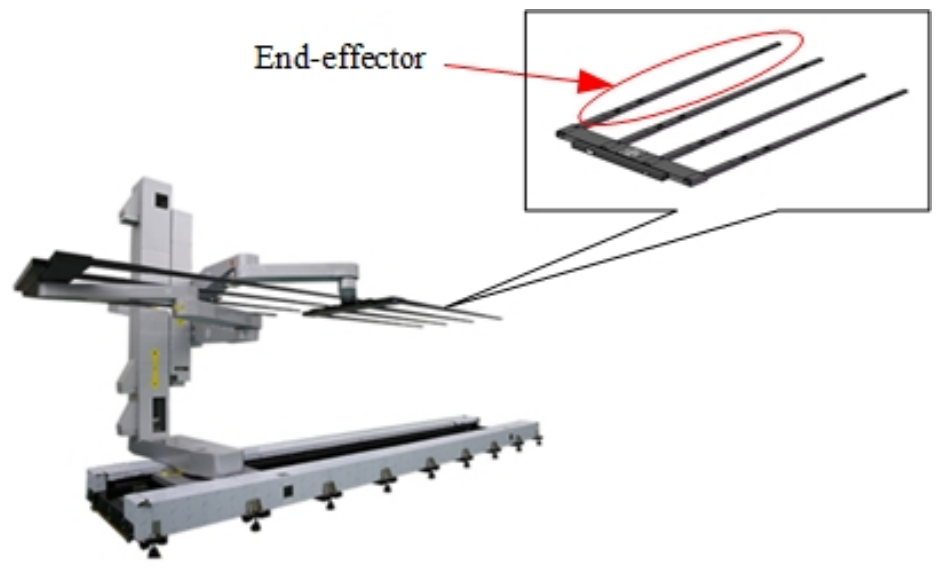

Figure 2. Structure of end-effector.

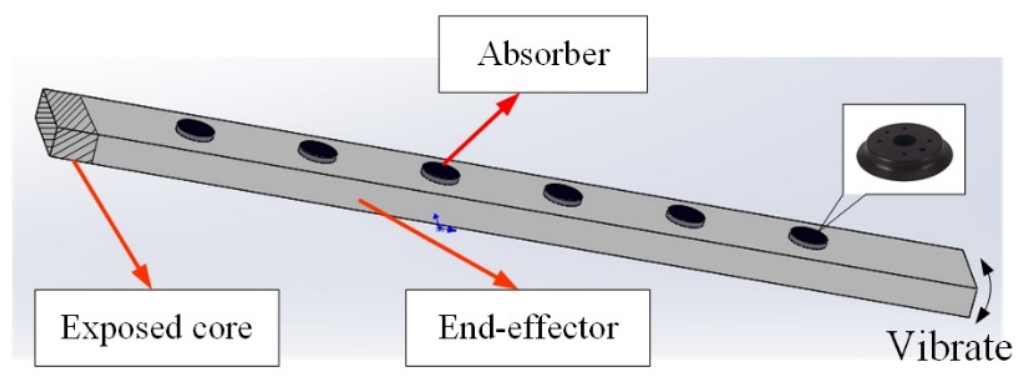

Figure 3. The model of end-effector.

From Figure 3, it can be found that the absorber is omitted after comparing the mass so that we can simplify the end-effector into a thin cantilever beam. According to the actual length, width, and height of the end-effector, we reduced in equal proportion. The structural parameters of the cantilever beam designed and processed for system identification are $250 \times 2 \times 25 \mathrm{in} \mathrm{mm}$. Additionally, on the left there are $30 \mathrm{~mm}$ left to fix the end which is called exposed core. The specific structure is shown in Figure 4 . The parameters of the pasted piezoelectric ceramics are shown in Table 1.

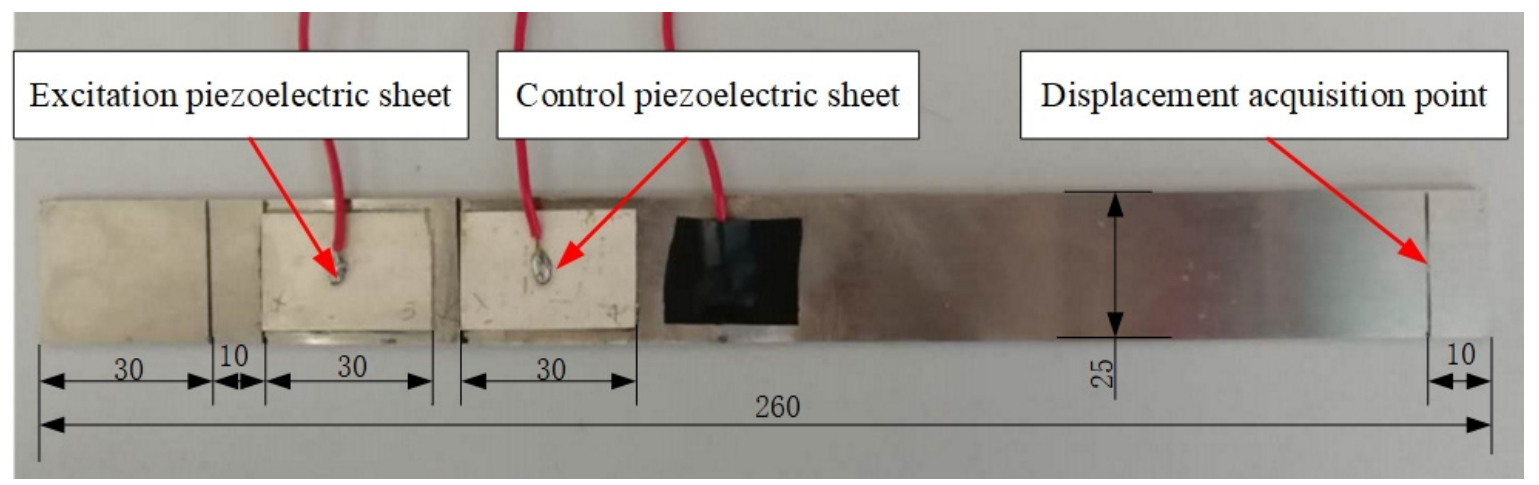

Figure 4. Structure of the cantilever beam. 
Table 1. The parameters of the pasted piezoelectric ceramic.

\begin{tabular}{ccc}
\hline Parameters & Symbol & Value \\
\hline Elastic constant $\left(\mathrm{N} / \mathrm{m}^{2}\right)$ & $\mathrm{C} 11$ & $12.6 \times 10^{10}$ \\
& $\mathrm{C} 12$ & $5.5 \times 10^{10}$ \\
& $\mathrm{C} 13$ & $5.3 \times 10^{10}$ \\
& $\mathrm{C} 33$ & $11.7 \times 10^{10}$ \\
Piezoelectric constant $\left(\mathrm{C} / \mathrm{m}^{2}\right)$ & $\mathrm{C} 44$ & $3.53 \times 10^{10}$ \\
& $\mathrm{e} 31$ & -6.5 \\
& $\mathrm{e} 33$ & 23.3 \\
Dielectric constant $(\mathrm{C} / \mathrm{Vm})$ & $\mathrm{e} 15$ & 17.0 \\
\hline
\end{tabular}

In this paper, the excited signal of the end-effector comes from the contacting end with the arm of the robot. In order to restore the excitation of the end-effector as realistically as possible, we pasted a piezoelectric sheet as close to the exposed core as we can in order to excite the vibration. The maximum strain of a cantilever beam is at the clamp, so we pasted the other piezoelectric sheet near the excited one to control the vibration. Expressing the actual output vibration characteristics of the system called displacement acquisition point is $10 \mathrm{~mm}$ relative to the other end. The response of $0-300 \mathrm{~Hz}$ sinusoidal sweep excitation signal of the cantilever beam is carried out by fast Fourier transform. As shown in Figure 5, the first-order natural frequency of the structure is about $32.8 \mathrm{~Hz}$, and the second-order natural frequency is about $195 \mathrm{~Hz}$. The two natural frequencies have a large distance on the horizontal axis. The frequency domain response amplitude difference is large too. Therefore, the first-order vibration mode of the structure is mainly considered in the identification process. Based on the structure of the cantilever beam, this paper designs the experiment shown in Figure 6.

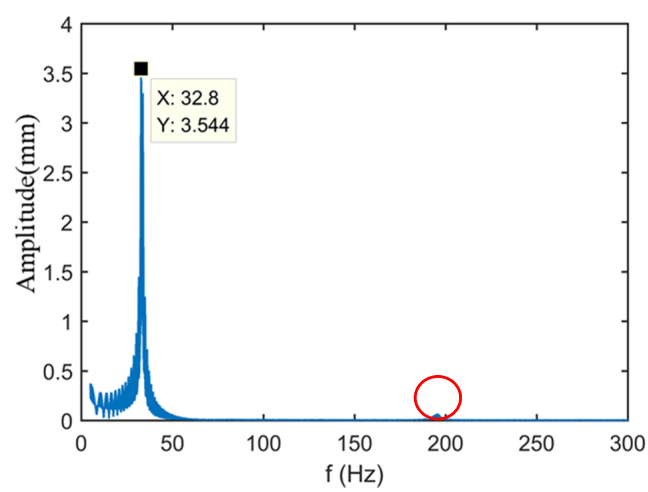

Figure 5. Single-side amplitude spectrum of cantilever structure.

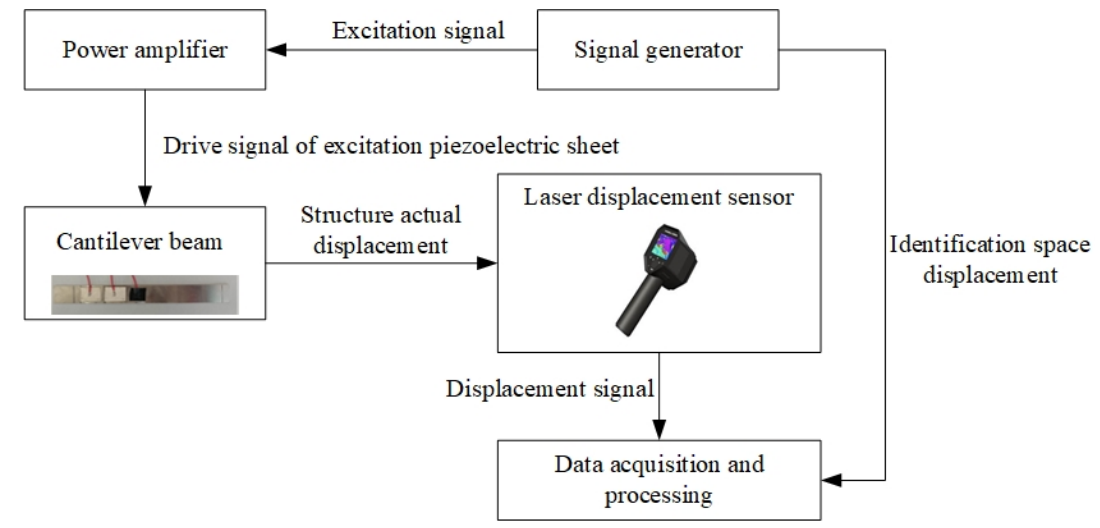

Figure 6. Experimental schematic diagram. 


\section{Linear System Identification}

\subsection{Theoretical Design of Linear System Identification}

Basing on the state space model of the system, the discrete state space model of linear time invariant system is assumed to be as follows,

$$
\begin{gathered}
x_{k+1}=A x_{k}+B u_{k}+w_{k} \\
y_{k}=C x_{k}+D u_{k}+v_{k}
\end{gathered}
$$

where:

$x_{k}$-the n-dimensional state vector of the system

$y_{k}$-the L-dimensional output observation vector

$u_{k}$-the m-dimensional input observation vector, $A \in R^{n \times m}, C \in R^{n \times l}, D \in R^{l \times m}$

$\omega_{k}$-the n-dimensional input noise vector

$v_{k}$-the L-dimensional measurement noise vector.

$[u(k), y(k)]$ 一the input-output sequence of SISO object at time $\mathrm{k}$

The parameter vector is defined as

$$
\theta=\left[\operatorname{vec}(A)^{T}, \operatorname{vec}(B, G)^{T}, \operatorname{vec}(C)^{T}, \operatorname{vec}(D)^{T}\right]^{T} \in R^{d}
$$

The vector length $d=n^{2}+n(m+l)+l m$ is the total number of system parameters. $v e c(A)$ is the straightening operation. The identification of system state space can be expressed as the estimate of the parameter matrix $A, B, C, D$ by giving the input-output observation sequence $\left(U_{N}, Y_{N}\right)$, so as to minimize the value of the objective function as

$$
J_{N}(\theta)=\sum_{k=1}^{n}\left\|y_{k}-\hat{y}_{k \mid k-1}(\theta)\right\|_{2}^{2}=\sum_{k=1}^{n}\left\|e_{k}(\theta)\right\|_{2}^{2}
$$

Basing on subspace identification method, the extended objective matrix of structure is proposed as

$$
\Gamma_{a}=\left[C C A \cdots C A^{a-1}\right]^{T}
$$

Hankel matrix of impulse response

$$
H_{t, a, b}=\left[\begin{array}{cccc}
h_{t} & h_{t+1} & L & h_{t+b-1} \\
h_{t+1} & h_{t+2} & L & h_{t+b} \\
M & M & M & M \\
h_{t+a-1} & h_{t+a} & L & h_{t+a+b-2}
\end{array}\right]
$$

According to the corresponding time, the Hankel matrix is $U_{p}=U_{1, s, T}$ in the past which at present is $U_{c}=U_{s, 1, T}$ and $U_{f}=U_{s+1, s, T}$ in the future. Hankel matrices defining the future measurement noise and input noise are denoted as $M_{f}, N_{f}$. The state sequence of a system is defined as

$$
X_{s}=\left[\begin{array}{llll}
x_{s+1} & x_{s+2} & \cdots & x_{s+T}
\end{array}\right]
$$

Basing on the input-output matrix equation of the system $Y_{f}=\Gamma_{s} X_{s}+\Phi_{s} U_{f}+\Phi_{s}{ }^{w} M_{f}+N_{f}$. Removing $M_{f}, N_{f}$ and Hankel matrices $U_{f}$ by projection method gets $O_{s}=\Gamma_{s} X_{s}$. 
Basing on the estimated state sequence $X_{s}$ and extended observability matrix $\Gamma_{s}$, The parameter matrix $A, B, C, D$ of the system state space model can be obtained by solving the equations with the least square method of N4SID regression.

$$
\left[\begin{array}{cc}
\hat{A} & \hat{B} \\
\hat{C} & \hat{D}
\end{array}\right]=\underset{A, B, C, D}{\operatorname{argmin} \|}\left[\begin{array}{c}
X_{S+1} \\
Y_{\mathcal{C}}
\end{array}\right]-\left[\begin{array}{cc}
A & B \\
C & D
\end{array}\right]\left[\begin{array}{l}
X_{s} \\
U_{\mathcal{C}}
\end{array}\right]_{F}^{2}
$$

\subsection{Linear System Identification Experiment}

System identification generally can be divided into off-line identification and on-line identification. In this paper, this system model structure and system order have been determined and the background is the industrial robot, so we used the off-line identification, as shown in Figure 7.

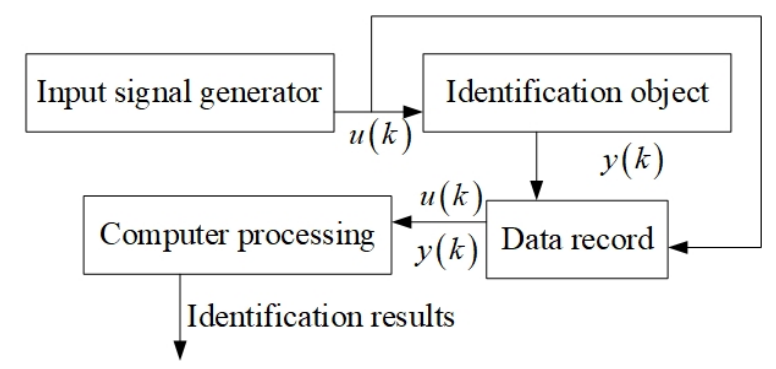

Figure 7. Structure diagram of off-line identification.

This paper mainly studied the low-frequency vibration of the cantilever beam so the experimental platform (illustrated as Figure 8) was built according to the cantilever beam model designed in Section 2. By amplifying the voltage by 30 times, the power amplifier excites the vibration of the cantilever structure. The laser displacement sensor gathers the displacement signal from the displacement acquisition point. In the process of the experiment, different excitation signals were used to excite the system vibration and collect the real-time signals.

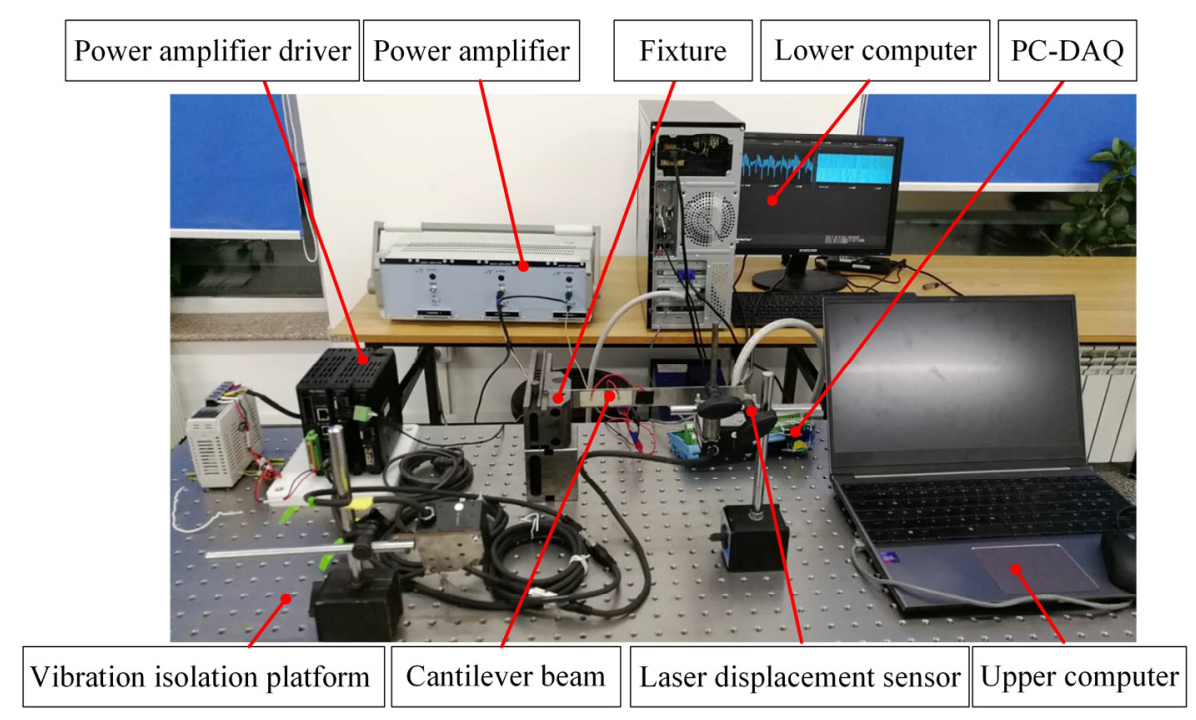

Figure 8. System identification experimental platform.

The order of state space identification is assumed to be 4 , and the identification results are presented in Table 2. The accuracy of the identification results is represented by the root mean square value (MSE) of the actual output value and the output value of the identification model. In the process of data acquisition, the laser displacement sensor is set in advance to carry out sliding average filtering 
with a length of 265 to reduce the influence of measurement noise. Because the output signal of a laser displacement sensor is the absolute value of its measured displacement value, it is necessary for the average and the trend of the output signal before identification. The identified system model is set as a discrete state space model.

Table 2. Results of different identification system.

\begin{tabular}{ccc}
\hline Excitation Signal & Sampling Frequency $\mathbf{( H z )}$ & Root Mean Square Error \\
\hline white Gaussian noise & 1000 & 33.6493 \\
& 500 & 6.2939 \\
PRBS sequence & 200 & 2.9043 \\
& 1000 & 29.2545 \\
& 500 & 15.7613 \\
$0-100 \mathrm{~Hz}$ Chirp Signal & 200 & 2.6542 \\
$0-200 \mathrm{~Hz}$ Chirp Signal & 1000 & 14.2563 \\
$0-300 \mathrm{~Hz}$ Chirp Signal & 1000 & 30.4611 \\
& 1000 & 26.1471 \\
\hline
\end{tabular}

In the above table it can be seen that the difference of the excitation signal and sampling frequency will have a significant impact on the identification results. In the above experiments, the optimal performance (i.e., minimum MSE) of Gaussian white noise, Pseudo-Random Binary Sequence (shorted as PRBS) sequence and chirp sweep signal are 2.9043, 2.6542 and 14.2563, respectively. The experiment should be repeated many times to avoid the interference of external factors. By comparing the output of actual and the identification space while three different kinds of signals excited the structure at different frequencies, the optimal situation under different excitation signals is selected. The time-domain differential value of the system is obtained by subtracting the actual output displacement of the system and the output displacement of the identification space at the corresponding time, as shown in Figure 9.

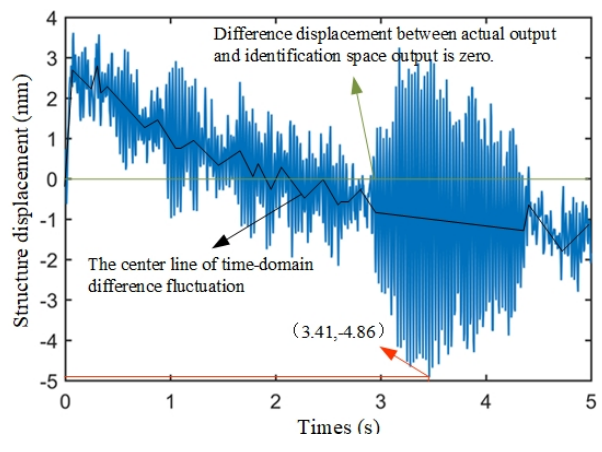

(a) Gaussian white noise at $200 \mathrm{~Hz}$

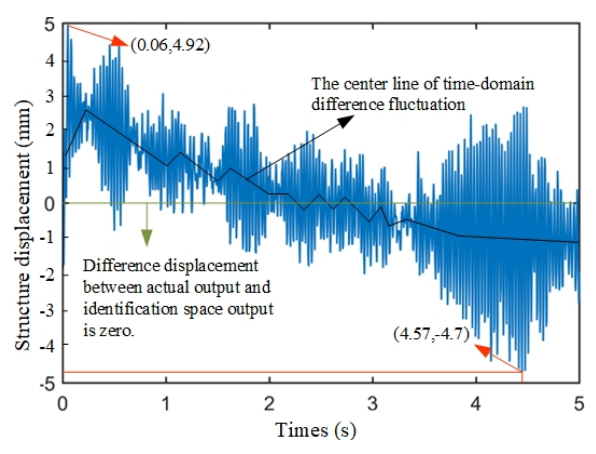

(b) PRBS sequence at $200 \mathrm{~Hz}$

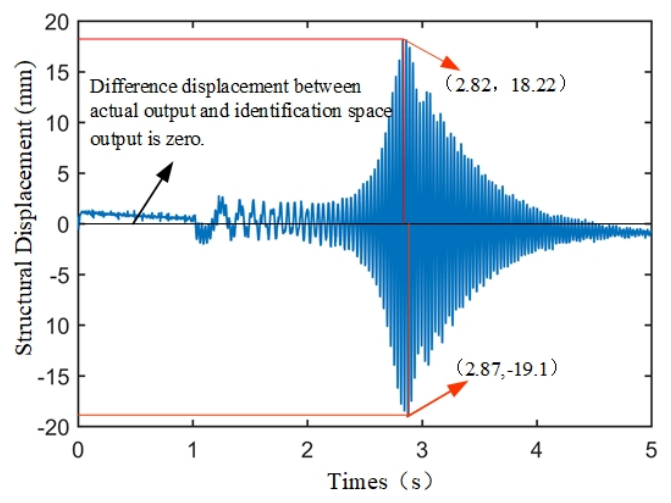

(c) $0-100 \mathrm{~Hz}$ Chirps sequence

Figure 9. Displacement difference between actual output and identification space output. 
Figure 9 shows that the influence of different excitation signals on the system identification can be directly represented by the time-domain differential curve. The experimental results show that in the whole system identification process, the maximum output displacement differences of Gaussian white noise, PRBS excitation signal and 1-100 Hz are $4.86 \mathrm{~mm}, 4.92 \mathrm{~mm}$ and $19.1 \mathrm{~mm}$, respectively. Although the values of Gaussian white noise. PRBS excitation signal are smaller than that of the chirps signal, the identification results of these two have the similar time-varying identification difference deviation. But the curve of $0-100 \mathrm{~Hz}$ Chirps is stable around line 0 and the maximum difference is focused on a very short time. Therefore, considering the accuracy of identification results, the coverage of frequency bandwidth and the real-time requirements of sampling frequency, this paper chooses 1-100 Hz Chirp signal as the final estimation of the mathematical model and excitation signal of controller. The final parameter matrix is as

$$
\begin{aligned}
& A=\left[\begin{array}{rrrr}
0.4933 & -0.8513 & 0.0474 & -0.0366 \\
0.8514 & 0.5098 & 0.0324 & -0.0139 \\
-0.1372 & 0.0714 & 0.3828 & 0.3508 \\
0.0797 & -0.0527 & 0.0799 & 0.8603
\end{array}\right] \\
& B=\left[\begin{array}{r}
-0.0061 \\
-0.0034 \\
-0.0288 \\
0.0157
\end{array}\right] \\
& C=[313.457-221.691-53.6114-4.8011] \\
& D=0
\end{aligned}
$$

\section{Design and Simulation of LQG Controller}

For the discrete state space, the link quality indicator regulator, abbreviated as LQI regulator, obtains the optimal control rate by minimizing the linear quadratic cost function $J(u)=\sum_{k=0}^{Y=}\left(z^{T} Q z+u^{T} R u\right)$ without considering the noise interference. $Q$ and $r$ are the weight matrix of cost function determined by human. While the control strategy of the linear-quadratic-Gaussian controller shorted as LQG controller is to minimize the error quadratic functional of linear systems disturbed by external noise. It does not need the system state to be fully observable. Therefore, it is more suitable for this control system to be calculated by adjusting the value of weight $Q$ and $r$ during designing. The state estimator gives the state equation as

$$
\hat{x}[k+1 \mid k]=A \hat{x}[k \mid k-1]+B u[k]+L(y[k]-C \hat{x}[k \mid k-1]-D u[k])
$$

According to the state estimation of Kalman filter and the optimal control matrix $\mathrm{K}$ obtained by the LQI method, a 1-DOF position tracking controller is constructed. The structure diagram is shown in Figure 10.

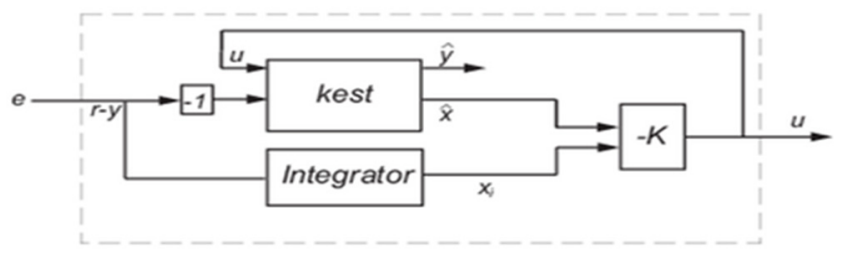

Figure 10. Linear-quadratic-Gaussian (LQG) controller block diagram.

Figure 11 illustrates the comparison of the Bode diagram between the identification system and the original system. The amplitude of the identification system is consistent with the amplitude of the Bode diagram of the actual input-output relationship between $0 \mathrm{~Hz}$ and $100 \mathrm{~Hz}$. But there is a huge 
deviation beyond this range. As for the phase, there is an understandable delay between $0 \mathrm{~Hz}$ and $100 \mathrm{~Hz}$, but there is no regular pattern during overstep.

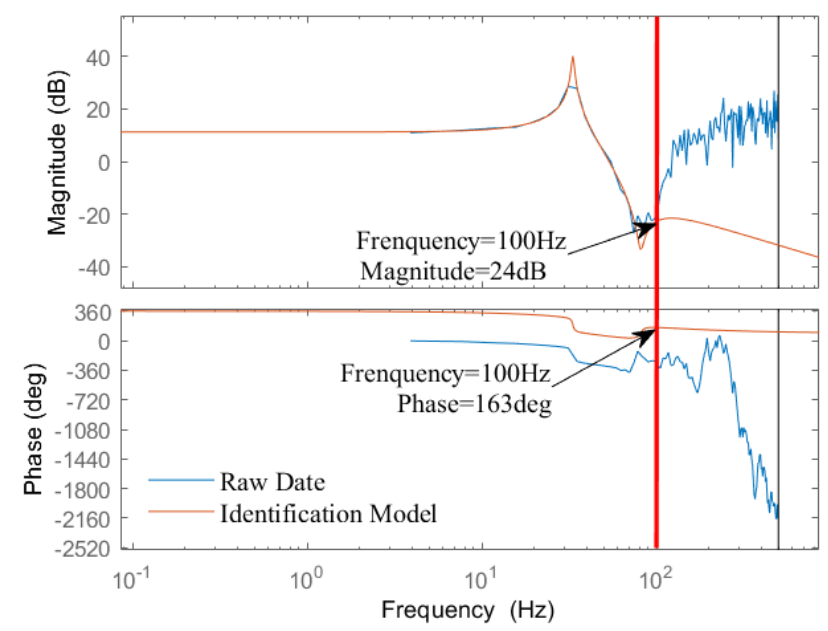

Figure 11. Comparison of Bode diagram between identification system and original system.

Figures 12 and 13 show the Bode diagram of the closed-loop system and the LQG controller. Generally, the Bode diagram amplitude of the closed-loop system is less than 1 and the phase diagram is basically maintained at 360 degrees when the frequency is below $100 \mathrm{~Hz}$ after adding the LQG controller, which indicates that the closed-loop system can effectively suppress the vibration below $38.7 \mathrm{~Hz}$.

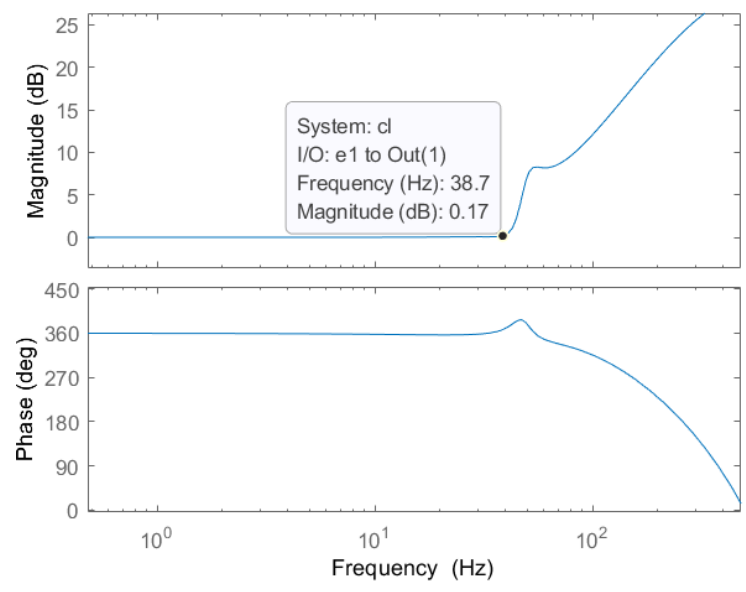

Figure 12. Bode diagram of closed-loop system.

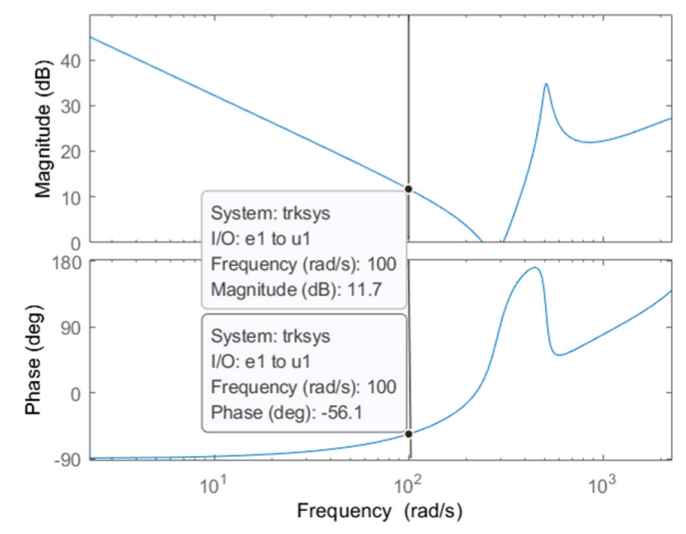

Figure 13. Bode diagram of LQG Controller. 
Figure 14 shows the results of the response for the open-loop system and the closed-loop system, respectively. It can be seen that the amplitude of the closed-loop system has a rapid reduction which means the dynamic performance of the system is significantly improved after adding the controller.

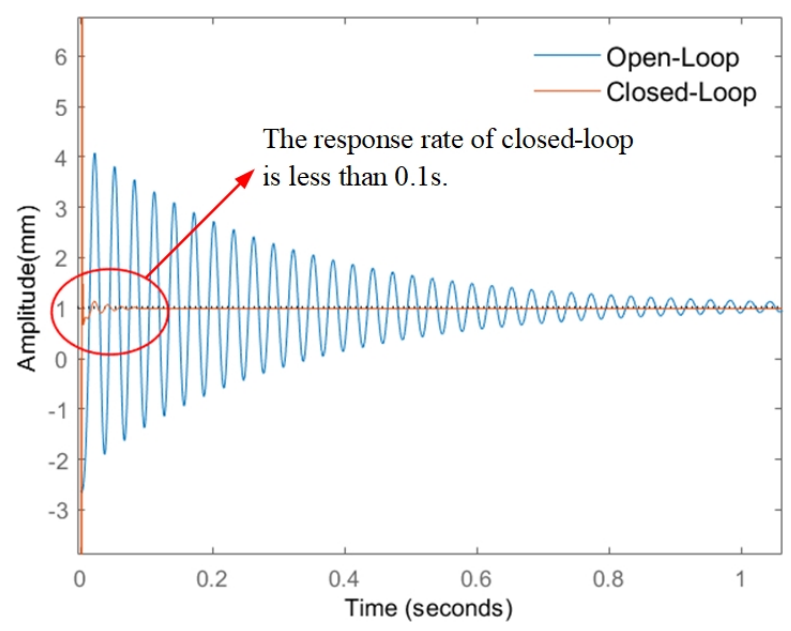

Figure 14. Response curve of open and closed loop systems.

The system controller designed by appeal is simulated. The frequency of sinusoidal interference signal is $35 \mathrm{~Hz}$ which is near the natural frequency, and the vibration amplitude is $\pm 20 \mu \mathrm{m}$. The vibration response of the structure is shown in Figure 15. It can be seen from Figure 15 that the vibration amplitude of the structure is successfully reduced from $\pm 20 \mu \mathrm{m}$ to $\pm 1 \mu \mathrm{m}$ after the effective suppression of the control system indicating that the vibration suppression percentage of the vibration suppression system is as high as $95 \%$ without high frequency noise. When the vibration frequency of the excitation signal is $50 \mathrm{~Hz}$ and the vibration amplitude is $\pm 20 \mu \mathrm{m}$, the response of the system reaches about $\pm 40 \mu \mathrm{m}$. The results are shown in Figure 16, and the control system completely loses the vibration suppression effect. In conclusion, the LQG vibration suppression system has an obvious suppression effect on vibration interference less than $38.7 \mathrm{~Hz}$, but has no suppression effect on other vibration signals higher than $38.7 \mathrm{~Hz}$.

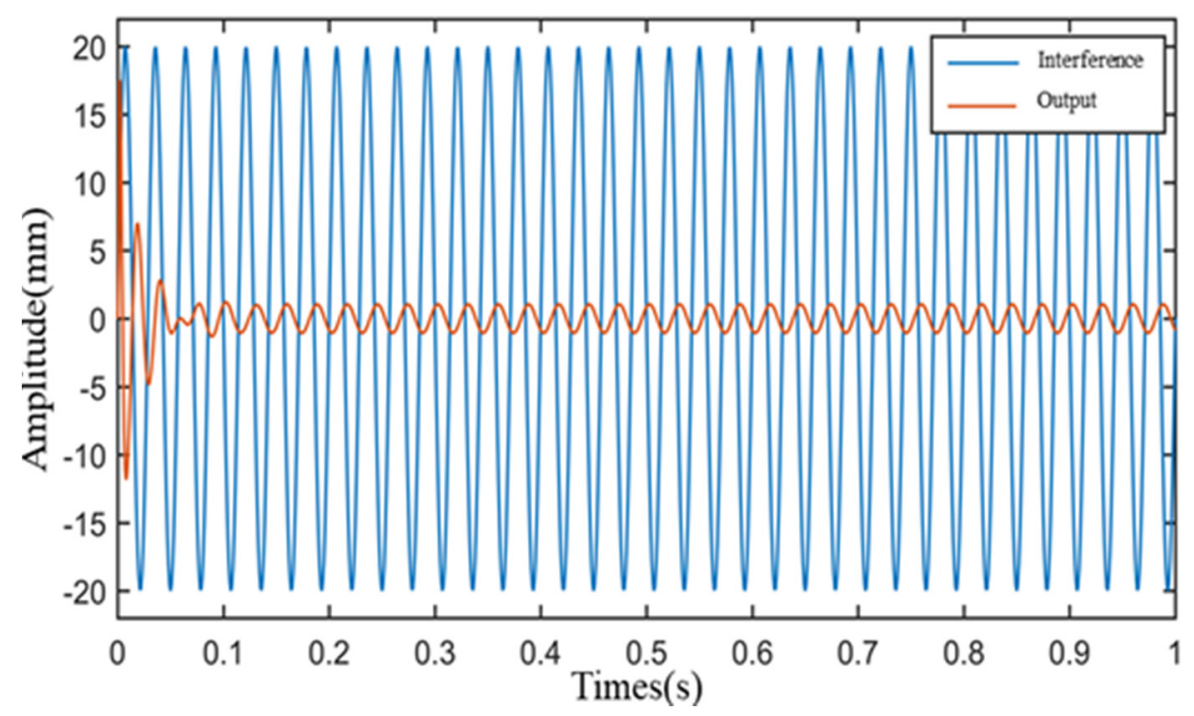

Figure 15. Simulation results of $35 \mathrm{~Hz}$ interference. 


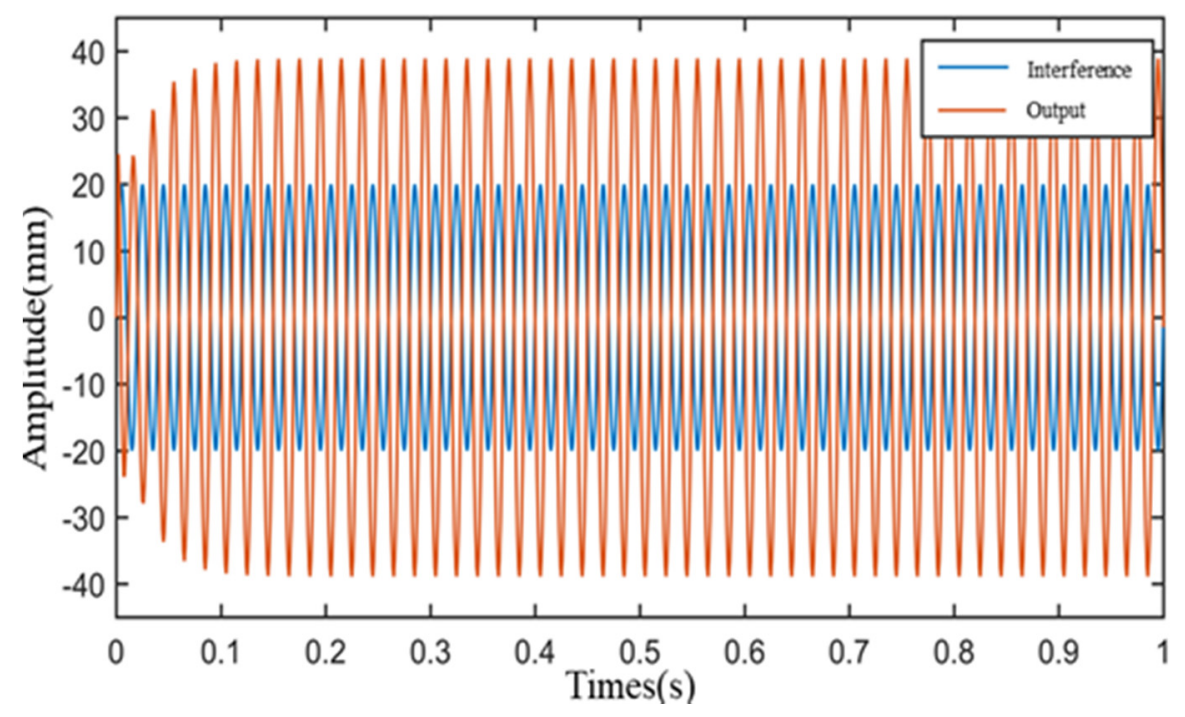

Figure 16. Simulation results of $50 \mathrm{~Hz}$ interference.

\section{Conclusions}

In this paper, the vibration control of a cantilever beam is discussed. In order to solve the low frequency vibration problem of industrial robot cantilever structure, a discrete state space mathematical model based on subspace identification method is proposed. Based on the model, an LQG controller is designed. The results of the simulation demonstrate that the LQG control method has a fast response speed while the frequency range is limited, so it requires high accuracy of the identification model. When the excitation frequency is lower than $38.7 \mathrm{~Hz}$, the LQG controller designed in this paper can effectively reduce the first-order frequency of the system and make the vibration amplitude reach $95 \%$ and the vibration amplitude of the structure is successfully reduced from $\pm 20 \mu \mathrm{m}$ to $\pm 1 \mu \mathrm{m}$.

This study provides a feasible method for the vibration control of the cantilever beam. The subspace identification method is used to identify the discrete state of the system, which is suitable for industrial transport robots with end-effectors. At the same time, the LQG controller designed based on the identification results can control the vibration of the end-effector of the robot without the real-time feedback signal of the system. This method can be implemented in vibration control of industrial robots with an end-effector.

Author Contributions: All the authors have made great contributions to the design of the system. X.S. and J.F. supervised the experimental measurements and the writing of the manuscript. H.S. designed and processed the structure, performed the experiment, analyzed the data and wrote the paper. C.Z. and G.W. provided valuable suggestions and comments on the interpretation of results and on the paper. All authors have read and agreed to the published version of the manuscript.

Funding: This work was supported by the National Key R\&D Program of China under Grant No. 2018 YFB1308500.

Conflicts of Interest: The authors declare no conflict of interest.

\section{References}

1. Li, Y.; Ge, S.S.; Wei, Q.; Gan, T.; Tao, X. An online trajectory planning method of a flexible-link manipulator aiming at vibration suppression. IEEE Access 2020, 8, 130616-130632. [CrossRef]

2. Botta, F.; Scorza, A.; Rossi, A. Optimal piezoelectric potential distribution for controlling multimode vibrations. Appl. Sci. 2018, 8, 551. [CrossRef]

3. Won, C.C.; Sulla, J.L.; Sparks, D.W., Jr.; Belvin, W.K. Application of piezoelectric devices to vibration suppression. J. Guid. Control. Dyn. 1994, 17, 6. [CrossRef]

4. Giurgiutiu, V. Review of smart-materials actuation solutions for aeroelastic and vibration control. J. Intell. Mater. Syst. Struct. 2000, 11, 525-544. [CrossRef] 
5. Crawley, E.F.; De Luis, J. Use of piezoelectric actuators as elements of intelligent structures. AIAA J. 1987, 25, 1373-1385. [CrossRef]

6. Tzou, H.S. Integrated distrubuted sensing and active vibration suppression of flexible manipulators using distrubuted piezoelectrics. J. Robot. Syst. 1989, 6, 745-767. [CrossRef]

7. Tzou, H.; Wan, G.; Tseng, C. Dynamics and distributed vibration controls of flexible manipulators; integrated distributed sensing and active piezoelectric actuator. In Proceedings of the 1989 International Conference on Robotics and Automation, Scottsdale, AZ, USA, 14-19 May 2003; Institute of Electrical and Electronics Engineers (IEEE): New York, NY, USA, 2003; pp. 1716-1721.

8. Shen, Z.; Liu, Y.; Zhao, J.; Tang, X.; Chen, W. Design and experiment of a small legged robot operated by the resonant vibrations of cantilever beams. IEEE Access 2017, 5, 8451-8458. [CrossRef]

9. Lin, C.-Y.; Jheng, H.-W. Active vibration suppression of a motor-driven piezoelectric smart structure using adaptive fuzzy sliding mode control and repetitive control. Appl. Sci. 2017, 7, 240. [CrossRef]

10. Lou, J.; Liao, J.; Wei, Y.; Yang, Y.; Li, G. Experimental identification and vibration control of a piezoelectric flexible manipulator using optimal multi-poles placement control. Appl. Sci. 2017, 7, 309. [CrossRef]

11. Leang, K.K.; Devasia, S. Feedback-linearized inverse feedforward for creep, hysteresis, and vibration compensation in AFM piezoactuators. IEEE Trans. Control. Syst. Technol. 2007, 15, 927-935. [CrossRef]

12. Zhang, $\mathrm{T}$. Influence of hysteresis on the vibration control of a smart beam with a piezoelectric actuator by the bouc-wen model. Shock. Vib. 2020, 2020,1-11. [CrossRef]

13. Chen, X.; Hisayama, T. Adaptive sliding-mode position control for piezo-actuated stage. IEEE Trans. Ind. Electron. 2008, 55, 3927-3934. [CrossRef]

14. Dadfarnia, M.; Jalili, N.; Xian, B.; Dawson, D.M. A lyapunov-based piezoelectric controller for flexible cartesian robot manipulators. J. Dyn. Syst. Meas. Control. 2004, 126, 347-358. [CrossRef]

15. Qiu, Z.-C.; Wu, H.-X.; Ye, C.-D. Acceleration sensors based modal identification and active vibration control of flexible smart cantilever plate. Aerosp. Sci. Technol. 2009, 13, 277-290. [CrossRef]

16. Chang, T.; Kwadzogah, R.; Caudill, R. Vibration control of linear robots using a piezoelectric actuator. IEEE/ASME Trans. Mechatron. 2003, 8, 439-445. [CrossRef]

17. Jia, S.; Jiang, Y.; Li, T.; Du, Y. Learning-based optimal desired compensation adaptive robust control for a flexure-based micro-motion manipulator. Appl. Sci. 2017, 7, 406. [CrossRef]

18. Zehetner, C.; Gerstmayr, J. Compensation of flexible vibrations in a two-link robot by piezoelectric actuation. Mech. Model Based Control Smart Mater. Struct. 2010, 205-214.

19. Douat, L.R.; Queinnec, I.; Garcia, G.; Michelin, M.; Pierrot, F.H. Infinity control applied to the vibration minimization of the parallel robot par2. In Proceedings of the 2011, IEEE International Conference on Control Applications, Denver, CO, USA, 28-30 September 2011; pp. 947-952.

20. Williams, D.; Khodoparast, H.H.; Yang, C. Active vibration control of a flexible link robot with the use of piezoelectric actuators. In Proceedings of the International Conference on Engineering Vibration, Sofia, Bulgaria, 4-7 September 2018.

21. Narendra, K.; Parthasarathy, K. Adaptive identification and control of dynamical systems using neural networks. In Proceedings of the 28th IEEE Conference on Decision and Control, Tampa, FL, USA, 13-15 December 2003.

22. Jalil, N.A.; Darus, I.Z.M.; Mohamad, M. Neuro-fuzzy identification of flexible beam structure. In Proceedings of the 2012 IEEE Conference on Control, Systems \& Industrial Informatics, Bandung, Indonesia, 23-26 September 2012.

23. Sethi, V.; Song, G.; Qiao, P. System identification and active vibration control of a composite I-beam using smart materials. Struct. Control. Heal. Monit. 2006, 13, 868-884. [CrossRef]

24. Sethi, V.; Song, G. Multimodal vibration control of a flexible structure using piezoceramic sensor and actuator. J. Intell. Mater. Syst. Struct. 2007, 19, 573-582. [CrossRef]

25. Sethi, V.; Franchek, M.A.; Song, G. Active multimodal vibration suppression of a flexible structure with piezoceramic sensor and actuator by using loop shaping. J. Vib. Control. 2011, 17, 1994-2006. [CrossRef]

26. Elsley, R.K. A learning architecture for control based on back-propagation neural networks. In Proceedings of the IEEE International Conference on Neural Networks, San Diego, CA, USA, 24-27 July 1980.

27. Song, J.K.; Washington, G. Neural network discrimination in intelligent vibration control. J. Intell. Mater. Syst. Struct. 2000, 11, 234-242. [CrossRef] 
28. Chen, Q.; Chen, S.; Sun, Q.; Long, X.; Xu, R. Research on parameter identification of nonlinear friction on cantilever beam. J. Vibroeng. 2014, 16, 913-924.

29. Chen, Q.; Wang, M.; Yan, H.; Ye, H.; Yang, G. An adaptive method for inertia force identification in cantilever under moving mass. J. Vibroeng. 2012, 14, 1052-1058.

30. Takawa, T.; Fukuda, T. Controller design for vibration of a smart CFRP composite beam based on the fuzzy model. Int. J. Veh. Des. 2003, 33, 115-127. [CrossRef]

31. Zhang, Y. Vision servo of industrial robot: A review. In Proceedings of the American Institute of Physics Conference Series, Xi'an, China, 18 April 2018.

Publisher's Note: MDPI stays neutral with regard to jurisdictional claims in published maps and institutional affiliations.

(C) 2020 by the authors. Licensee MDPI, Basel, Switzerland. This article is an open access article distributed under the terms and conditions of the Creative Commons Attribution (CC BY) license (http://creativecommons.org/licenses/by/4.0/). 